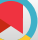

\title{
Prevalence of Chronic Orofacial Pain in Elderly Patients Referred to Shiraz Dental School From 2005 to 2017
}

\author{
Reza Derafshi ${ }^{1}$, Fahimeh Rezazadeh ${ }^{2}$, Janan Ghapanchi ${ }^{3,}{ }^{*}$, Delaram Basandeh Sharif ${ }^{4}$ and Mitra \\ Farzin $^{1}$ \\ ${ }^{1}$ Department of Prosthodontics, School of Dentistry, Shiraz University of Medical Sciences, Shiraz, Iran \\ ${ }^{2}$ Department of Oral and Maxillofacial Medicine, Oral and Dental Disease Research Center, School of Dentistry, Shiraz University of Medical Sciences, Shiraz, Iran \\ ${ }^{3}$ Department of Oral and Maxillofacial Medicine, School of Dentistry, Shiraz University of Medical Sciences, Shiraz, Iran \\ ${ }^{4}$ Undergraduate Student, Student Research Committee, School of Dentistry, Shiraz University of Medical Sciences, Shiraz, Iran \\ "Corresponding author: Department of Oral and Maxillofacial Medicine, School of Dentistry, Shiraz University of Medical Sciences, Shiraz, Iran. Email: ghabanj@sums.ac.ir
}

Received 2019 March 03; Revised 2019 October 02; Accepted 2019 October 24

\begin{abstract}
Background: Orofacial pain is presented mainly as a pain in the front part of the head, including the oral cavity. Adult and elderly patients are important target groups affected by this chronic pain, which may cause several problems.

Methods: In this cross-sectional study, the characteristics of patients who suffered from orofacial pain were evaluated. The data such as patients' gender, age, pain diagnoses, treatments, responses to treatments, duration and severity of pain, and disability were collected from their records. The diagnoses were provided based on the characteristics of pain, X-ray findings, physiological findings, lab tests, nerve block injection, and a drug challenge test.

Results: Among all the 5,221 patients referred to Oral and Maxillofacial Medicine and Prosthodontics Department, 583 (11.16\%) reported orofacial pain as the chief complaint. The frequency of orofacial pain was more prevalent among women than men. Burning mouth syndrome was the most prevalent cause of orofacial pain in the elderly, followed by temporomandibular disorder and trigeminal neuralgia.

Conclusions: Most cases of orofacial pain in elderly patients were burning mouth syndrome, temporomandibular disorder, and neuralgia. It was more observed in females than males with the mean age of $55.3 \pm 10.7$ years old.
\end{abstract}

Keywords: Orofacial Pain, Elderly Patient, Chronic Pain, Prevalence

\section{Background}

Orofacial pain (OFP) is presented mainly as a pain in the front part of the head, including the oral cavity. The origin of the pain may be musculoskeletal, dental, neural, or paranasal sinuses (1). Orofacial pain is referred to as pains in the face (under eye up to the neck) and mouth (2). In some cases, appropriate diagnosis may be overlooked or delayed, resulting in unsuitable management and sustained symptoms (1).

The trigeminal nerve is the largest sensory nerve in the human body, defending the vital organs that support our survival (brain, eyes, nose, mouth). Unsurprisingly, discomfort in the trigeminal system is often irresistible and inevitable for the affected individual (3). Chronic pain is determined as pain staying for more than 12 weeks. Otherwise, it may be related to autoimmune illness or central/peripheral sensitization. In older individuals, neuropathy or arthritis are the main cause of chronic pain (4). The prevalence of OFP varies from $1.3 \%$ to $48.4 \%$. Some stud- ies on OFP have shown joblessness and diminished work efficacy among affected patients (5).

Trigeminal neuralgia (TN) is a long-lasting pain triggering the trigeminal nerve. There are two main forms: typical and atypical. The typical type causes periods of intensive, unexpected, shock-like pain in the affected side of the face that continues for seconds to a few minutes. The atypical type makes a continuous burning pain that is not very severe. Episodes may be activated by any touch to the face. In some cases, both types may occur in the same case. It is one of the most long-lasting painful disorders that can cause depression. Burning mouth syndrome (BMS) is an undesirable feeling in the mouth with no primary dental or medical source. No associated signs of illness are distinguished in the oral cavity (6).

Temporomandibular joint dysfunction (TMD) is a type of pain and impairment of masticatory muscles and temporomandibular joints. The most significant finding is pain, followed by limited mandibular movement $(7,8)$. 


\section{Objectives}

Few studies have evaluated chronic orofacial pain in elderly patients, especially in Iran. Therefore, this study was conducted to evaluate the prevalence of chronic facial pain in elderly patients referred to Shiraz Dental School from 2005 to 2017.

\section{Methods}

The study was a cross-sectional study aimed to evaluate the prevalence of orofacial pain in elderly subjects referred to the Shiraz Oral Medicine and Prosthodontics Department(Iran) during the past 12 years from October 2005 to August 2017, retrospectively. The local Ethics Committee of Shiraz University of Medical Science approved this research. The informed consent was obtained from all patients. The patient file included sex, age, medical and dental history, medicine usage, clinical examination, and a detailed history of the pain duration, condition and symptom. A thorough history of synchronism of dental procedure, parafunctional habits or some psychoemotional disorders (depression or anxiety) and pain onset was obtained. For a definitive diagnosis, other than obtaining a complete history, where appropriate, laboratory procedures, radiography and drug challenge tests were also performed. Incomplete patients' records and undiagnosed subjects were excluded from the study. The OFP generally includes four main groups: temporomandibular disorders (TMD), neuralgia, psychological facial pain, and others (headache, odontogenic, sinusitis, etc.) based on the International Association for the Study of Pain (IASP) criteria. Pain severity at the base time and recall sessions was assessed by the Visual Analogue scale (VAS) and categorized to mild ( 1 - 4), moderate ( 5 - 7), and severe ( 8 - 10). Response to treatment also was described as complete remission/significant improvement, partial response, and no response.

\subsection{Statistical Analysis}

Data were analyzed using the SPSS software (SPSS, version 22, Hong Kong) using descriptive statistical methods (means, standard deviations, median and percentages) and chi-square test. The level of significance was $\alpha=0.05$.

\section{Results}

From 5,221 patients' files of Oral Medicine and Prosthodontics Department, 583 (11.16\%) OFP files were gathered of which 39 were incomplete or undiagnosed.

Demographic data included 328 (56.26\%) women and 255 (43.73\%) men, with the mean age of $55.3 \pm 10.7$ years (age range: 46 - 90). The frequency of OFP was more prevalent among women than men. Burning mouth syndrome was the most prevalent cause of OFP in adult and elderly subjects ( 267 cases $=45.7 \%$ ), followed by TMD (188 cases $=32.3 \%$ ) and trigeminal neuralgia ( 98 cases $=16.80 \%$ ), sinusitis (17 cases $=2.9 \%$ ), odontogenic referral pain (12 cases $=2.05 \%)$ and traumatic neuroma in 1 case $(0.17 \%)$ of all the patients. Postherpetic neuralgia was found in 3 cases (0.51\%), and multiple sclerosis-related neuralgia was found in $2(0.34 \%)$ subjects (Table 1$)$. Older and retired subjects showed more OFP than others. The pain onset duration varied from 11 days to 3.4 years. The prevalence of OFP in people with the mean age of $46 \pm 11.2$ was higher than that in individuals at other ages. A significant association was found between gender and orofacial pain $(\mathrm{P}=0.001), \mathrm{OR}=$ 1.77 (45\% CI: $-1.45-2.16$ ). Moreover, $29 \%$ of the patients had a history of dental treatment, especially tooth extraction and root canal therapy, before pain onset. In $44.3 \%$ of the subjects, psychoemotional disorders (depression or anxiety) were reported and of whom $21 \%$ had experienced a stressful condition before their pain onset (Table 2).

\begin{tabular}{ll}
\hline Table 1. The Number and Percentage of OFP in Patients & \\
\hline OFP & Values \\
\hline Burning mouth syndrome (BMS) & $267(45.7)$ \\
\hline Temporomandibular disorders (TMD) & $188(32.3)$ \\
\hline Trigeminal neuralgia & $98(16.8)$ \\
\hline Headache sinusitis & $17(2.9)$ \\
\hline Odontogenic referral pain & $12(2.05)$ \\
\hline Traumatic neuroma & $1(0.17)$ \\
\hline Post herpetic neuralgia & $3(0.51)$ \\
\hline Multiple sclerosis related neuralgia & $2(0.34)$ \\
\hline
\end{tabular}

Abbreviation: OFP, orofacial pain

${ }^{\mathrm{a}}$ Values are expressed as No. (\%).

In BMS patients, emotional instability was found in a large group (54.4\%), followed by systemic diseases (17.8\%). No etiologic causes were detected in $10.3 \%$ of the patients, and 7.8\% suffered from psychological disorders. The tongue was the most frequent location of burning (45.2\%), followed by palate and buccal mucosa. Idiopathic burning sensations were mostly founded in old age cases.

A large number of TMD patients had a history of parafunctional habits such as cheek biting, bruxism, clenching, addiction and smoking. The most common treatment modality for TMD was pharmacotherapy (73.3\%), occlusal appliance (14.2\%) and physiotherapy (21.7\%), respectively. In addition, for 45 patients (16.8\%), dental treatment was advised. A total of 53 cases (19.8\%) referred to neurologists and psychiatrists.

The most common treatment modality for OFP was 


\begin{tabular}{lccc}
\hline Table 2. Descriptive Data of Different OFP Forms & & & TMD \\
\hline OFP & BMS & Pharmacotherapy & Neuralgia \\
\hline Treatment modality & Pharmacotherapy & 43 & Pharmacotherapy \\
Response to treatment, \% & 17.3 & Parafunctional habits \\
Etiologic factor & Emotional instability & 11 days \\
Pain onset duration & 4 months & No \\
\hline
\end{tabular}

Abbreviations: BMS, burning mouth syndrome; OFP, orofacial pain; TMD, temporomandibular joint dysfunction.

pharmacotherapy. For BMS, it included antidepressant (like amitriptyline), anticonvulsant (like gabapentin) and topical clonazepam. In TMD, the most common type of medicine was muscle relaxants (like methocarbamol) and NSAIDs (like naproxen). Anticonvulsant (like carbamazepine) was also the common medicine for neuralgia. Complete pain relief was reported in $78.4 \%$ of neuralgias, $43 \%$ of TMDs, and $17.3 \%$ of psychogenic pain. Most of the patients with OFP had severe pain (62.1\%). Patients with neuralgia had the most severe pain, while patients with TMD reported the mildest pain severity. Pain-related life disabilities such as insomnia, limitation of mouth opening, chewing, speaking, and inability for routine activities were observed in $33.7 \%$ of our patients.

\section{Discussion}

The orofacial pain prevalence in elderly patients was $11.16 \%$. To the best of our knowledge, there are few research studies into different aspects of facial pain in elderly patients. This was more than the prevalence of OFP in the general population in Zakrzewska's study (7\%) (9). This difference may be related to case selection as we evaluated OFP only in older age patients. The most common type of OFP in elderly patients was BMS (45.7\%). This result is in agreement with previous studies (10). However, in other studies, TMD (11), neuropathic pain $(12,13)$ or cluster headache were demonstrated to have a higher rate than others. This difference may be related to patient selection and the type of chronic pain evaluated.

Different facial pains are more common in females than in males, which are consistent with our results. It seems that women mostly seek treatment of pain. Tomoyasu et al. and Lipton et al. achieved the same results in their study. Aging is a risk factor for OFP, therefore, the elderly people would need better evaluation and management of pain. It has been reported $(14,15)$ that neuropathic pain is more common in older patients, which is consistent with our results. It is also in agreement with the result of another study reporting a higher incidence of chronic OFP by increasing age (16). Thus it is necessary to concentrate on non-odontogenic chronic OFP in these patients.
During the time, changes in all parts of the body (somatic and emotional) are unavoidable. However, various pathologic processes in the elderly are one of the important risk factors of disorders (17). It is believed that depression is the most predominant mental health problem in older age cases. Depression can atypically be the cause of somatic symptoms like fatigue $(18,19)$. Then again, chronic pain and depression are closely associated with each other in elderly patients. Chronic pain is prevalent in the elderly, and it can affect emotional components (20).

An epidemiological study demonstrated that depression was approximately 2.5 and 4.1 times more than other problems in chronic pain $(21,22)$. Likewise, major depressive disorders are at a higher risk (three times more than nociceptive pain and six times more than neuropathic pain) (23). These documents suggested that long-lasting inflammation in nerve could signify both chronic pain and depression. In chronic pain, peripheral injury causes inflammation in neurons. An impaired relation between glia and mast cells also results in poor neuronal cell function $(24,25)$. Previous studies reported that $13 \%$ of old individuals were affected by both depression and chronic pain and the coexistence of both was not unusual (26).

Our limitations were the number of samples in each category of OFP, and no data about psychological status or quality of life. Thus, it is recommended that further studies with better and precise methods on a larger sample size should be conducted.

\subsection{Conclusions}

The most prevalent forms of OFP in elderly patients were BMS, TMD, and neuralgia. It occurred more in females than males with the mean age of $55.3 \pm 10.7$ years old. Dentists should refer the elderly patients with orofacial pain to specialized oral clinics for better evaluation before becoming chronic and making several unnecessary procedures.

\section{Footnotes}

Authors' Contribution: Janan Ghapanchi: study design and concept, data interpretation, drafting, final approval, and agreement to be accountable for all aspects of the 
work. Delaram Basandeh Sharif: study design and concept, data collection, drafting, final approval, and agreement to be accountable for all aspects of the work. Reza Derafshi: data interpretation, drafting, final approval, and agreement to be accountable for all aspects of the work. Mitra Farzin: data interpretation, drafting, final approval, and agreement to be accountable for all aspects of the work. Fahimeh Rezazadeh: study design, data interpretation, drafting, final approval, and agreement to be accountable for all aspects of the work.

Conflict of Interests: The authors declared that there was no conflict of interest.

Ethical Approval: The local Ethics Committee of Shiraz University of Medical Science approved this research.

Funding/Support: Shiraz University of Medical Science supported this study financially.

Informed Consent: The patient provided informed consent for the publication of this report.

\section{References}

1. Gur A, Sarac AJ, Cevik R, Altindag O, Sarac S. Efficacy of $904 \mathrm{~nm}$ gallium arsenide low level laser therapy in the management of chronic myofascial pain in the neck: A double-blind and randomize-controlled trial. Lasers Surg Med. 2004;35(3):229-35. doi: 10.1002/lsm.20082. [PubMed: 15389743].

2. Brisman R. Gamma knife surgery with a dose of 75 to 76.8 Gray for trigeminal neuralgia. I Neurosurg. 2004;100(5):848-54. doi: 10.3171/jns.2004.100.5.0848. [PubMed: 15137604].

3. Alfredo PP, Bjordal JM, Dreyer SH, Meneses SR, Zaguetti G, Ovanessian $\mathrm{V}$, et al. Efficacy of low level laser therapy associated with exercises in knee osteoarthritis: A randomized double-blind study. Clin Rehabil. 2012;26(6):523-33. doi: 10.1177/0269215511425962. [PubMed: 22169831].

4. Brisman R. Trigeminal neuralgia: Diagnosis and treatment. World Neurosurg. 2011;76(6):533-4. doi:10.1016/j.wneu.2011.06.028. [PubMed: 22251500].

5. Zhang $\mathrm{H}$, Lei D, You C, Mao BY, Wu B, Fang Y. The long-term outcome predictors of pure microvascular decompression for primary trigeminal neuralgia. World Neurosurg. 2013;79(5-6):756-62. doi: 10.1016/j.wneu.2012.01.040. [PubMed: 22381868].

6. Jang $\mathrm{H}$, Lee $\mathrm{H}$. Meta-analysis of pain relief effects by laser irradiation on joint areas. Photomed Laser Surg. 2012;30(8):405-17. doi: 10.1089/pho.2012.3240. [PubMed: 22747309]. [PubMed Central: PMC3412059].

7. Jiang JA, Chang WD, Wu JH, Lai PT, Lin HY. Low-level laser treatment relieves pain and neurological symptoms in patients with carpal tunnel syndrome. J Phys Ther Sci. 2011;23(4):661-5. doi:10.1589/jpts.23.661.

8. Rezazadeh F, Hajian K, Shahidi S, Piroozi S. Comparison of the effects of transcutaneous electrical nerve stimulation and low-level laser therapy on drug-resistant temporomandibular disorders. I Dent (Shiraz). 2017;18(3):187-92. [PubMed: 29034273]. [PubMed Central: PMC5634358].

9. Konstantinovic LM, Cutovic MR, Milovanovic AN, Jovic SJ, Dragin AS, Letic M, et al. Low-level laser therapy for acute neck pain with radiculopathy: A double-blind placebo-controlled randomized study. Pain
Med.2010;11(8):1169-78. doi:10.1111/j.1526-4637.2010.00907.x. [PubMed: 20704667].

10. Shakur SF, Bhansali A, Mian AY, Rosseau GL. Neurosurgical treatment of trigeminal neuralgia. Dis Mon. 2011;57(10):570-82. doi 10.1016/j.disamonth.2011.09.003. [PubMed: 22036113].

11. Rezazadeh F, Rahimi S. Evaluation of chronic orofacial pain in dental patients; a 10 years retrospective study. Asian J Med Pharm Res. 2017;7(1):1-5.

12. Koopman JS, Dieleman JP, Huygen FJ, de Mos M, Martin CG, Sturkenboom MC. Incidence of facial pain in the general population. Pain. 2009;147(1-3):122-7. doi: 10.1016/j.pain.2009.08.023. [PubMed: 19783099].

13. Moore K. Lasers and pain treatment. Laser Part Clinix. 2004;72:1-6.

14. Kumar A, Brennan MT. Differential diagnosis of orofacial pain and temporomandibular disorder. Dent Clin North Am. 2013;57(3):419-28. doi: 10.1016/j.cden.2013.04.003. [PubMed: 23809301].

15. Ghapanchi J, Bazargani A, Zariean A, Rezaee M, Ranjbar Z, Karami A et al. Evaluation of the anti-Streptococcus mutans potential of Petroselinum crispum, an in vitro Study. Eur J Med Plants. 2016;15(4):1-8 doi: 10.9734/ejmp/2016/27095.

16. Hegedus B, Viharos L, Gervain M, Galfi M. The effect of lowlevel laser in knee osteoarthritis: A double-blind, randomized, placebo-controlled trial. Photomed Laser Surg. 2009;27(4):577-84. doi: 10.1089/pho.2008.2297. [PubMed: 19530911]. [PubMed Central: PMC2957068].

17. Niccoli T, Partridge L. Ageing as a risk factor for disease. Curr Biol. 2012;22(17):R741-52. doi: 10.1016/j.cub.2012.07.024. [PubMed: 22975005].

18. Anderson DN. Treating depression in old age: the reasons to be positive. Age Ageing. 2001;30(1):13-7. doi: 10.1093/ageing/30.1.13. [PubMed: 11322666].

19. Smith OR, Kupper N, Schiffer AA, Denollet J. Somatic depression predicts mortality in chronic heart failure: Can this be explained by covarying symptoms of fatigue? Psychosom Med.2012;74(5):459-63. doi: 10.1097/PSY.ob013e31824ef2f4. [PubMed: 22511727].

20. Sykioti P, Zis P, Vadalouca A, Siafaka I, Argyra E, Bouhassira D, et al. Validation of the Greek version of the DN4 diagnostic questionnaire for neuropathic pain. Pain Pract. 2015;15(7):627-32. doi: 10.1111/papr.12221. [PubMed: 24796220].

21. McCarthy LH, Bigal ME, Katz M, Derby C, Lipton RB. Chronic pain and obesity in elderly people: results from the Einstein aging study. $J$ Am Geriatr Soc. 2009;57(1):115-9. doi: 10.1111/j.1532-5415.2008.02089.x. [PubMed: 19054178]. [PubMed Central: PMC2763486].

22. Bauer H, Emeny RT, Baumert J, Ladwig KH. Resilience moderates the association between chronic pain and depressive symptoms in the elderly. Eur J Pain. 2016;20(8):1253-65. doi: 10.1002/ejp.850. [PubMed: 26914727].

23. Ohayon MM, Stingl JC. Prevalence and comorbidity of chronic pain in the German general population.J Psychiatr Res. 2012;46(4):444-50. doi: 10.1016/j.jpsychires.2012.01.001. [PubMed: 22265888].

24. Ji RR, Xu ZZ, Gao YJ. Emerging targets in neuroinflammationdriven chronic pain. Nat Rev Drug Discov. 2014;13(7):533-48. doi: 10.1038/nrd4334. [PubMed: 24948120]. [PubMed Central: PMC4228377].

25. Giustino V. Chronic pain in the elderly: The case for new therapeutic strategies. Pain Physician. 2015;18(E863):E863-76.

26. Mossey JM, Gallagher RM. The longitudinal occurrence and impact of comorbid chronic pain and chronic depression over two years in continuing care retirement community residents. Pain Med. 2004;5(4):335-48. doi: 10.1111/j.1526-4637.2004.04041.x. [PubMed: 15563319]. 